\title{
Tailoring mTOR-based therapy: molecular evidence and clinical challenges
}

The mTOR signaling pathway integrates inputs from a variety of upstream stimuli to regulate diverse cellular processes including proliferation, growth, survival, motility, autophagy, protein synthesis and metabolism. The mTOR pathway is dysregulated in a number of human pathologies including cancer, diabetes, obesity, autoimmune disorders, neurological disease and aging. Ongoing clinical trials testing mTOR-targeted treatments number in the hundreds and underscore its therapeutic potential. To date mTOR inhibitors are clinically approved to prevent organ rejection, to inhibit restenosis after angioplasty, and to treat several advanced cancers. In this review we discuss the continuously evolving field of mTOR pharmacogenomics, as well as highlight the emerging efforts in identifying diagnostic and prognostic markers, including miRNAs, in order to assess successful therapeutic responses.

KEYWORDS: biomarkers cancer cardiovascular disease clinical trials dual inhibitors FKBP12 microRNA mTOR rapamycin resistance

\section{The history of mTOR}

In the 1960s, the natural macrocyclic lactone rapamycin $\left(\mathrm{C}_{51} \mathrm{H}_{79} \mathrm{NO}_{13}\right.$; molecular weight: 914.2), also known as sirolimus, was obtained from the bacterium Streptomyces hygroscopicus found in soil samples from Rapa Nui (Easter Island). In the 1970s, rapamycin was used as a potent antifungal agent and shortly afterwards was found to inhibit cell proliferation and possess strong immunosuppressive properties [1,2]. It took 20 years to identify the molecular target of rapamycin and to elucidate its mechanism of action. This was achieved by selection of spontaneous mutations that confer resistance to the growth inhibitory effect of rapamycin in the budding yeast Saccharomyces cerevisiae. Three genes were identified. The first was $R B P 1$, a homolog of the human peptidyl-prolyl isomerase FKBP12, which was identified using an FK506 binding column in a search for receptors for the immunosuppressant drug with structural homology to rapamycin [3]. Deletion of RBP1 in yeast resulted in recessive drug resistance while expression of human FKBP12 restored rapamycin sensitivity [4]. Therefore, rapamycin forms a toxic complex with FKBP12 inhibiting the function of other cellular proteins [4]. The other two genes were $T O R 1$ and $T O R 2$, originally called $D R R 1$ and $D R R 2$, which encode two highly homologous proteins $(\sim 280 \mathrm{kDa})[5,6]$.

Soon after, mTOR was identified [7]. Unlike yeast, higher order eukaryotes have only one TOR gene. mTOR is a large $(289 \mathrm{kDa})$ serine/threonine protein kinase that belongs to the phosphatidylinositol 3'-kinase-related kinase (PIKK) family. At the C-terminus it contains a PIKK domain that exhibits serine and threonine kinase activity but not lipid kinase activity as seen with the other PIKK family members [8]. Another unique characteristic of mTOR, which does not exist in the other members of the PIKK family, is the $11 \mathrm{kDa}$ FKBP12-rapamycinbinding domain (FRB) at the $\mathrm{N}$-terminus of the PIKK domain $[9,10]$.

\section{Multiprotein complexes of mTOR}

Over the last 30 years mTOR-related research has provided significant insights into its molecular architecture and function. Today, it is clear that mTOR forms two distinct multiprotein complexes: mTORC1 and mTORC2. Both complexes are composed of mTOR, mLST8/ G $\mu \mathrm{L}$, Pras40, Deptor and Tti1/Tel2. Raptor and Pras 40 are specific to mTORC1, while Rictor, Protor $1 / 2$ and $m S i n 1$ are specific to mTORC2 (Box 1) [11].

In response to growth factors, stress, amino acids and oxygen, mTORC1 regulates numerous pathways including cell growth, autophagy, protein synthesis, cell cycle and metabolism. The best-known mTORC1 substrates are S6K1 and $4 \mathrm{E}-\mathrm{BP} 1$, both of which are involved in the regulation of protein synthesis. Importantly, S6K1 was shown to form a negative feedback loop by inhibiting expression of the insulin receptor substrate, attenuating the PI3K/Akt pathway (FIGURE 1) [12,13].

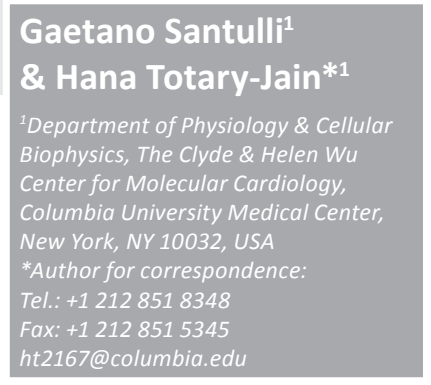

Future
Medicine $\mathrm{fS}$ 


Box 1. mTORC1 and mTORC2
complexes.
mTORC1 (specific)
" Raptor
- Pras40
mTORC1 and mTORC2
- mTOR
- Deptor
- mLst8
- Tti1/Tel2
mTORC2 (specific)
- Rictor
- Protor $1 / 2$
- mSin1

In contrast to mTORC1, little was known about mTORC2 until an important discovery demonstrated that mTORC2 is able to regulate cell proliferation, survival and metabolism by phosphorylating Akt on a key activation site $\left(\mathrm{Ser}^{473}\right)[14,15]$. Notably, Akt activates mTORC1 by phosphorylating and inhibiting the tuberous sclerosis complex, which negatively regulates mTORC1 by inhibiting the small GTPase Rheb that in turn directly activates mTORC1 (Figure 1) [16].

Dysregulation of the mTOR pathway has been linked to several pathological conditions, including cancer, diabetes, obesity, autoimmune disorders, neurological disease and aging, making it an attractive target for the development of new pharmacological treatments $[11,17,18]$.

\section{Rapamycin: mechanism of action \& first applications}

Upon entering the cell, rapamycin binds to FKBP12, which then interacts with the FRB domain of mTORC1 inhibiting its serine/threonine kinase activity and preventing the translation of specific cell cycle mRNAs required for G1 to $S$ phase transition, ultimately leading to growth arrest $[19,20]$. By contrast, the rapamycin-FKBP12 complex cannot interact with the FRB domain of mTORC2, and therefore cannot inhibit it acutely. In some cases, chronic treatment with rapamycin has been shown to block the mTORC2 assembly, thereby inhibiting its activity [21].

In 1999, rapamycin was approved by the US FDA as an immune suppressor agent to prevent organ rejection in renal transplant patients. Shortly thereafter, a number of studies demonstrated that rapamycin was also a potent inhibitor of vascular smooth muscle proliferation and migration [19,22-24]. These findings led to the development of the rapamycin-eluting stent used to treat atherosclerotic blockages in coronary arteries, which was approved by the FDA in 2003 to prevent in-stent restenosis. The antiproliferative properties of rapamycin were also tested in a broad range of malignancies and numerous clinical trials have been conducted.

\section{First-generation inhibitors of mTOR}

After the discovery of rapamycin and the elucidation of its mechanism of action, other macrolide ester derivatives (also known as 'rapalogs') that share the same mechanisms of action have been synthesized both to improve pharmacokinetic properties and to ensure patent protection (Figure 2). These include everolimus (RAD001) marketed by Novartis; temsirolimus (CCI-779) developed by Wyeth Pharmaceuticals; and deforolimus or ridaforolimus (AP23573) codeveloped by Merck and ARIAD Pharmaceuticals.

\section{Everolimus (RAD001)}

Everolimus is an oral rapamycin analog (halflife: $30 \mathrm{~h}$ ). It was approved by the FDA for the treatment of patients with advanced kidney cancer [25]; for the treatment of subependymal giant cell astrocytoma, alone or associated with tuberous sclerosis complex [26-28]; for progressive neuroendocrine tumors of pancreatic origin in patients with tumors not surgically removable; and for advanced hormone receptor-positive, HER2-negative breast cancer in combination with exemestane, after failure of treatment with anastrozole or letrozole [29]. Recently, the addition of everolimus to trastuzumab and vinorelbine was shown to extend progression-free survival in women with HER2positive advanced breast cancer, compared with treatment with placebo plus trastuzumab and vinorelbine, in the Phase III BOLERO-3 trial. The study enrolled women whose disease was resistant to prior trastuzumab treatment (Data presented at American Society of Clinical Oncology [ASCO] Meeting, June 2013 [30]). Everolimus is also used for the prevention of organ rejection after renal or heart transplants and in drug eluting coronary stents (Xience). The drug is being tested as a therapy for prostate, colorectal and non-small-cell lung cancers as either a single agent or in combination with additional therapies.

\section{Temsirolimus (CCl-779)}

Temsirolimus is a water-soluble ester derivative of rapamycin available in oral and intravenous 
formulations (half-life: 13-22 h). In 2007, it was approved by the FDA for the treatment of advanced-stage renal cell carcinoma [31]. It represents the first mTOR inhibitor approved for cancer therapy. Temsirolimus was also approved in Europe for the treatment of mantle cell lymphoma [32]. Temsirolimus is currently being tested either alone or in combination therapy in tumor types such as melanoma, myeloma, and renal and gynecological cancers.

\section{Deforolimus or ridaforolimus (AP23573)}

Ridaforolimus is an oral rapamycin analog (halflife: 56-74 h) that selectively targets mTOR and has been shown to possess potent antitumor activity both in cell lines and in xenograft tumor models. It is currently being evaluated (Phase III) in non-small-cell lung cancer, glioblastoma, soft tissue and bone sarcoma, prostate, endometrial and pancreatic cancer, metastastic breast cancer and relapsed hematological tumors. It has thus far shown good tolerability with dose-limiting stomatitis in both single and combination treatments. In November 2012, Merck officially withdrew its application for a marketing authorization for deforolimus concerning the maintenance treatment of patients with metastatic soft tissue sarcoma or bone sarcoma previously treated with chemotherapy owing to inconclusive benefit-risk balance evaluations.

\section{Clinical challenges}

Rapalogs are generally well-tolerated and do not lead to changes in blood pressure, liver function or renal toxicity, although fatigue, rash, hematological abnormalities and gastrointestinal disturbances are quite common. In addition, hypercholesterolemia and hypertriglyceridemia have also been reported; therefore, the monitoring of blood lipid levels is recommended [33].

The mTOR-targeted therapies are currently being evaluated in 1444 clinical trials (clinicaltrials.gov [101]) as single agent or in combination therapies. In preclinical studies performed in vitro and in vivo they have shown significant antiproliferative activity against a broad panel of tumors, with encouraging safety profiles and clinical benefit responses, achieving disease stabilization and/or tumor regression owing to inhibition of tumor cell proliferation. Notably, inhibition of the mTOR pathway also exerts antiangiogenic effects, mainly attributable to the fact that mTOR controls the production of HIF1 $\alpha$, which in turn mediates the expression of several angiogenic genes [34]. However, despite the proven efficacy of rapalogs against a number of tumors, their anticancer activity is quite unpredictable [35].

The negative feedback loop that exists downstream of mTORC1 clearly contributes to the

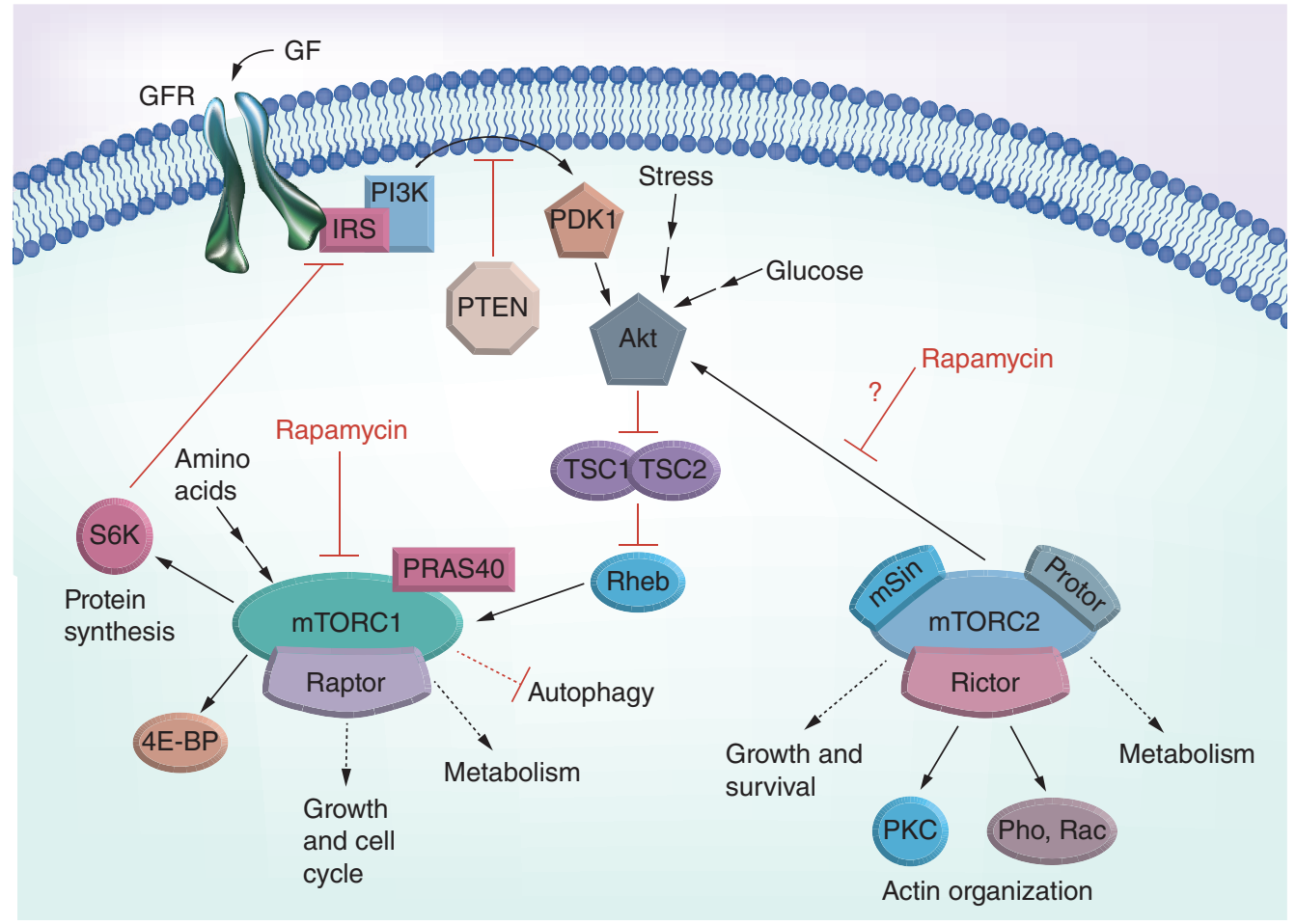

Figure 1. mTOR signaling pathways.

GF: Growth factor; GFR: Growth factor receptor; TSC: Tuberous sclerosis complex. 


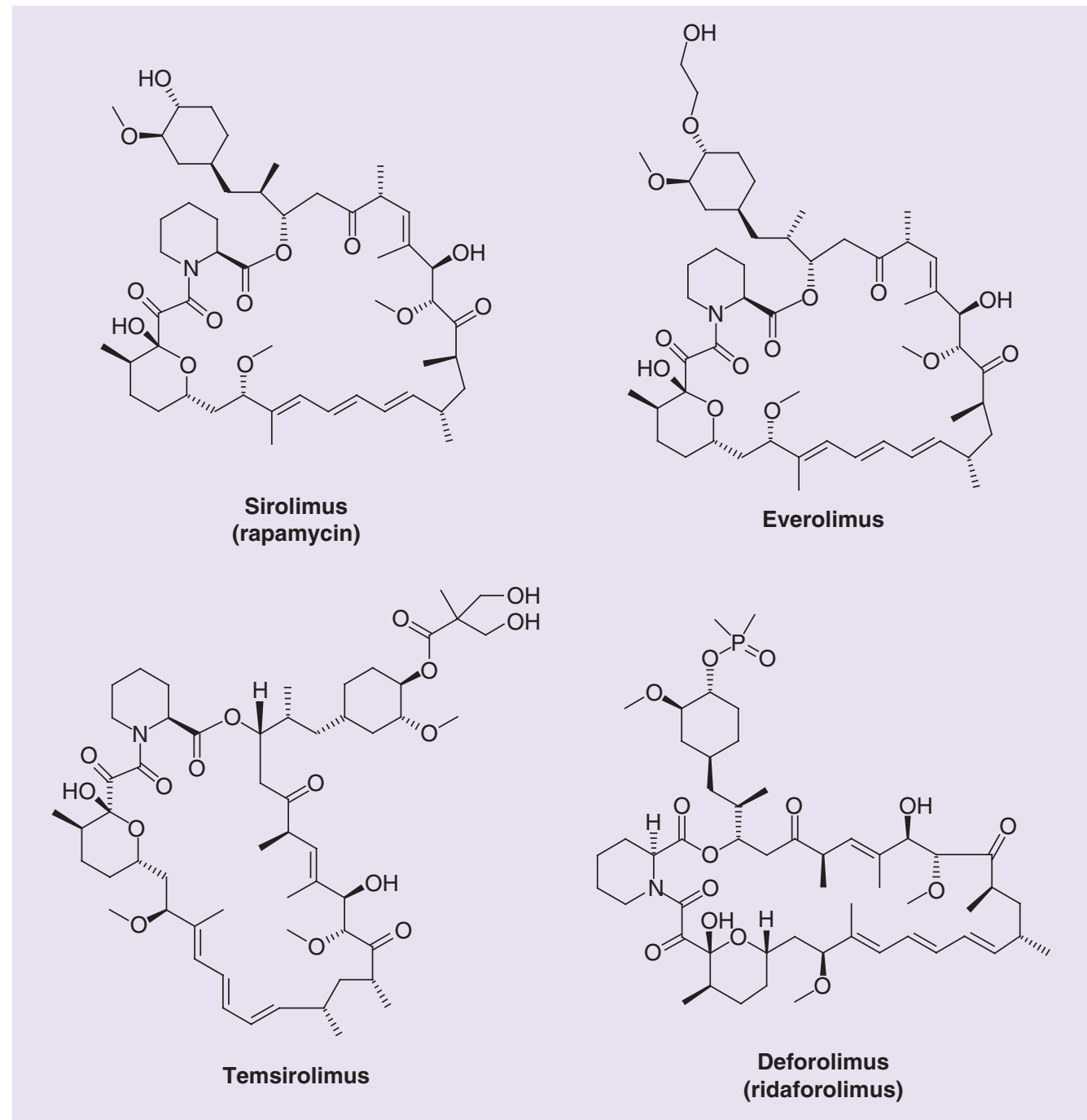

Figure 2. Molecular structures of rapamycin and the first generation of mTOR inhibitors (rapalogs).

observed resistance to rapalogs. Since active mTORC1 suppresses the PI3K/Akt pathway, mTORC1 inhibition by rapalogs abolishes the negative feedback loop, resulting in hyperactivation of the PI3K/Akt signaling and leading to increased cell survival (Figure 1) [36]. Notably, rapamycin-insensitive functions of mTORC1 were recently revealed, challenging the dogma that rapamycin completely inhibits mTORC1 activity [37,38]. Alternative survival pathways and crosstalk with other signaling pathways including MEK/ERK could also limit the efficacy of rapalogs [39]. In human cancers, inhibition of mTORC1 leads to MAPK pathway activation through a PI3K-dependent feedback loop [40]. Indeed, the combination of temsirolimus with the MAPK inhibitor, SL327, significantly reduced brain metastases in vivo, while treatment with temsirolimus alone yielded no significant effect [41].

\section{Second-generation inhibitors of mTOR}

New drugs, referred to as mTOR kinase domain inhibitors, are being developed to inhibit the ATP binding site of both mTORC1 and mTORC2. These drugs are small molecules that bind competitively and reversibly to the mTOR-ATP binding pocket, blocking the enzymatic activity of the kinase. Numerous mTORC1 and mTORC2 inhibitors are under preclinical evaluation and in Phase I/II clinical trials for various cancers (Table 1). Although mTOR kinase inhibitors target both complexes, preclinical and early clinical data showed hyperactivation of the PI3K/Akt signaling caused by decreased mTORC1 activity, which superseded the effects of inhibition of mTORC2.

Since the catalytic domain of mTOR and the p110 $\alpha$ subunit of PI3K are highly homologous, some second-generation compounds have dual 
activity against both PI3K and mTOR [42]. The main advantage of such dual inhibitors is the simultaneous inhibition of PI3K-Akt-mTOR signaling and reduction of the hyperactivation of PI3K that typically results in mTORC1 inhibition. Numerous dual PI3K/mTOR inhibitors have already entered Phase I and II clinical trials for a variety of cancer types, either alone or in combination with other chemotherapies (TABLE 1). Early clinical results suggest that these dual $\mathrm{PI} 3 \mathrm{~K} / \mathrm{mTOR}$ inhibitors are more efficacious than rapalogs, but also demonstrate increased toxicity. This was especially evident in the digestive tract with adverse effects including diarrhea, nausea and vomiting. Hyperglycemia has also been reported.

\section{Molecular biomarkers for mTOR-targeted therapy}

Our knowledge of the mTOR pathway has increased dramatically in recent years, yet many gaps still exist in our understanding of the molecular mechanisms involved in the response of cancer cells to such inhibitors. Therefore, there is an urgent need for efficient biomarkers not only to predict who will benefit from mTOR-targeted therapies, but also for patients to avoid developing unnecessary toxicities. In recent years, determinants of rapalog sensitivity and resistance have begun to emerge [43]. Several preclinical and clinical models showed that cancer cells where the mTOR pathway is hyperactive owing to PTEN deficiency [44],
Akt phosphorylation [45] or PI3K mutations [39] are particularly sensitive to mTOR inhibitors. Tumor cells that have functional apoptotic pathways, overexpress cyclin D1 [32,46], or have greatly increased angiogenic signaling [47] are more sensitive to rapamycin. Overexpression of antiapoptotic proteins such as $\mathrm{Bcl} 2$ may also serve as viable predictors of resistance to $\mathrm{mTOR}$ inhibitors [48]. Recently, we showed that rapamycin treatment was effective primarily in tumors that exhibit rapamycin-sensitive dephosphorylation of Akt and consequently decreased expression of the oncogene Skp2 [49]. However, these and many others candidate biomarkers still need to be validated in the clinical setting.

\section{miRNAs \& mTOR}

miRNAs are small ncRNAs (22 nucleotides) that regulate gene expression at the posttranscriptional level by either translational repression or mRNA cleavage [50]. miRNAs play an essential role in tumorigenesis, and it has recently been shown that circulating miRNAs can be measured in the serum and are emerging as a novel class of biomarkers for both diagnostic and prognostic assessments, giving rise to the field of miRNA pharmacogenomics [51,52].

Recently, various studies showed that miRNAs affect the mTOR pathway by targeting upstream regulators including IGF-R, PI3K, PTEN and Akt. Here we will focus on the functional role of miRNAs in the cellular response to mTOR inhibitors and the reciprocal regulation of mTOR

\section{Table 1. Second-generation mTOR and PI3K inhibitors currently in clinical trials} according to clinicaltrials.gov.

\begin{tabular}{|c|c|c|c|c|}
\hline Inhibitor & Targets & Status & Tumor & Ref. \\
\hline OSI-027 & mTORC1/mTORC2 & Phase I & Solid tumors & {$[74,75]$} \\
\hline Palomid 529 & mTORC1/mTORC2 & Phase I & Macular degeneration & [76] \\
\hline AZD8055 & mTORC1/mTORC2 & Phase I & Multiple cancers & {$[77-80]$} \\
\hline INK 128 & mTORC1/mTORC2 & Phase I & Solid tumors & [81] \\
\hline AZD2014 & mTORC1/mTORC2 & Phase I & Solid tumors & [82] \\
\hline CC-223 & mTORC1/mTORC2 & Phase I & Solid tumors & \\
\hline CC-115 & mTORC1/mTORC2 & Phase I & Solid tumors & \\
\hline GSK1059615 & $\mathrm{PI3K} / \mathrm{mTORC} 1 / \mathrm{mTORC2}$ & Phase I & Multiple cancers & [83] \\
\hline PF-05212384 (PKI-587) & $\mathrm{PI} 3 \mathrm{~K} / \mathrm{mTORC} 1 / \mathrm{mTORC} 2$ & Phase I & Solid tumors & [84] \\
\hline XL765 (SAR245409) & $\mathrm{PI3K} / \mathrm{mTORC} 1 / \mathrm{mTORC2}$ & Phase I & Solid tumors & [85] \\
\hline PF-04691502 & $\mathrm{PI3K} / \mathrm{mTORC} 1 / \mathrm{mTORC2}$ & Phase I & Solid tumors & [86] \\
\hline DS-7423 & $\mathrm{PI3K} / \mathrm{mTORC} 1 / \mathrm{mTORC2}$ & Phase I & Solid tumors & \\
\hline NVP-BEZ235 & $\mathrm{PI3K} / \mathrm{mTORC} 1 / \mathrm{mTORC2}$ & Phase I/II & Multiple cancers & [87-90] \\
\hline GDC-0980 & $\mathrm{PI3K} / \mathrm{mTORC} 1 / \mathrm{mTORC} 2$ & Phase I/II & Multiple cancers & [91] \\
\hline
\end{tabular}


and miRNAs (Figure 3). We demonstrated that rapamycin-resistant myogenic cells, developed by prolonged rapamycin treatment, displayed an extensive reprogramming of the miRNA transcriptome, characterized by upregulation of the oncogenic miR-17-92 and related clusters and downregulation of tumor suppressor miRNAs, such as miR-143, miR-29 and miR-22. Conversely, rapamycin-sensitive cells exhibited an increase in tumor suppressor miRNAs. Intriguingly, inhibition of members of the miR-17-92 cluster or delivery of tumor suppressor miRNAs restored sensitivity to rapamycin $[53,54]$. In support of our findings, rapamycin was shown to increase expression of miR-143 in human glioma cells [55]. Additionally, everolimus increased miR-143 expression in a time-dependent manner in human pancreatic cancer cells [56]. Endothelial cells treated with rapamycin showed increased levels of miR-21, mediating the antiproliferative and antimigration effect of rapamycin [57]. Moreover, mTOR was shown to control MyoD-dependent transcription of miR-1 in differentiating myoblasts and in mouse regenerating skeletal muscle [58]. Another report demonstrated that mTOR, by negatively controlling miR-125b biogenesis, regulates the production of IGF-II, a master switch governing the initiation of skeletal myogenesis [59].

On the other hand, several miRNAs, including miR-7a and miR-99a, were shown to directly target different components of the mTOR pathway [60-63]. Inhibition of miR-7a activated mTOR signaling and promoted adult $\beta$-cell replication in primary islets, which was reversed by rapamycin treatment [63]. In oral squamous cell

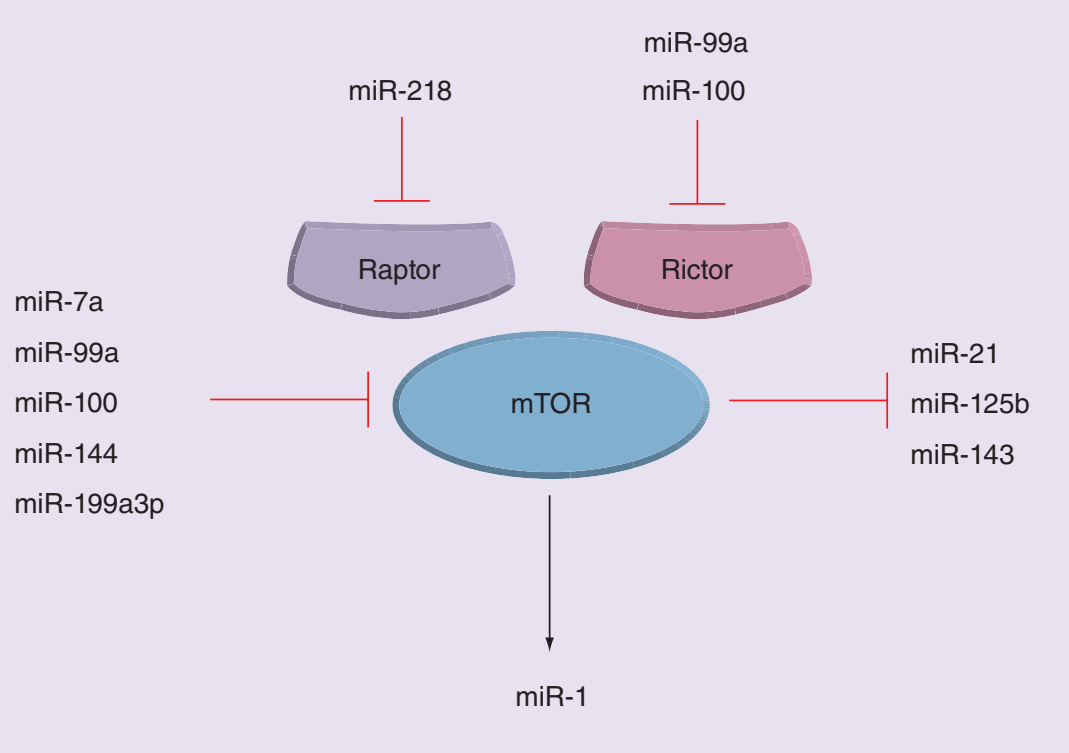

Figure 3. Model of the reciprocal regulation of mTOR and miRNAs. carcinoma and renal cell carcinoma, miR-99a was shown to directly modulate mTOR signaling pathways [61,62]. miR-99a downregulation was also associated with mTOR upregulation in various human lung cancer cells/tissues [64]. Interestingly, downregulation of miR-99a has been detected in different human cancers in which tumor growth was mediated by the upregulation of the tyrosine kinase c-Src [64]. Re-expression of miR-99a suppressed tumor growth of c-Srctransformed cells, and this effect was restored by mTOR overexpression. Downregulation of miR-99a was also observed in EGF- and Rastransformed cells, and was suppressed by inhibiting the MAPK pathway [64]. Additionally, in colorectal cancer, miR-144 downregulation led to poor prognosis via activation of the mTOR signaling pathway [65]. In oral squamous cell carcinoma, miR-218 targeted Rictor lead to an inhibition of Akt phosphorylation at $\operatorname{Ser}^{473}$ [66]. miR-100 was shown to repress mTOR signaling in endothelial and vascular smooth muscle cells, displaying an antiangiogenic function. Whereas miR-100 inhibition increased mTOR levels in endothelial cells, overexpression of miR-100 reduced mTOR expression and consequently attenuated cellular proliferation [67]. In clear-cell ovarian carcinoma cell lines, mTOR was repressed by miR-100 at the mRNA and protein levels, and decreased phosphorylation of 4EBP1 and p70 S6 kinase were also observed [68]. Human cytomegalovirus infection altered the expression of miR-100 and miR-101 to target components of the mTOR pathway, resulting in an improved ability to replicate [69]. In childhood adrenocortical tumors, miR-99a and miR-100 coordinately regulated the expression of mTOR, Raptor, and IGF via binding sites in their 3'-UTR regions [70]. In esophageal squamous cell carcinoma, wherein decreases in miR-99a and miR-100 are correlated with worse overall survival, miR-99a and miR-100 suppressed the expression of mTOR by directly binding to its 3'-UTR [71]. miR-101 was also shown to target mTOR in anaplastic large-cell lymphoma [72]. In a different study, miR-199a-3p, which is known to be downregulated in several human malignancies and in hepatocellular carcinoma, was also shown to target mTOR. The inhibition of miR-199a-3p in hepatocellular carcinoma cells led to G(1)-phase cell cycle arrest [73].

\section{Future perspective}

Our knowledge about the mTOR pathways has seen extraordinary advances over the past 30 years. The ever-expanding appreciation of 
the vastly versatile roles this molecule plays in cellular processes makes it enormously significant in human physiology and pathophysiology. Despite the challenges, mTOR-targeted therapies remain very attractive and promising. It is necessary to design future clinical trials to include validation of potential biomarkers. Research focusing on this particular area of investigation will certainly produce a plethora of invaluable information with regards to mTOR pharmacogenetics and pharmacogenomics, contributing to the advancement of drug discovery for the treatment of various human disorders in which the mTOR signaling pathway is involved. Eventually, novel therapeutic targets are likely to emerge downstream of mTOR with less toxicity, improved specificity and greater clinical benefits. Recent evidence describing how miRNAs regulate mTOR pathways will provide the framework for their development as diagnostic and prognostic biomarkers as well as for the design of novel therapeutic tools in the clinic.

\section{Acknowledgements}

The authors thank AR Marks, BW Osborne and T Lorberbaum (Columbia University) for editing the manuscript and for graphical assistance.

\section{Financial \& competing interests disclosure}

$G$ Santulli is supported by the American Heart Association, 13POST16810041 and H Totary-Jain is supported by the NHLBI/NIH, K99HL109133. The authors have no other relevant affiliations or financial involvement with any organization or entity with a financial interest in or financial conflict with the subject matter or materials discussed in the manuscript apart from those disclosed.

No writing assistance was utilized in the production of this manuscript.

\section{Executive summary}

\section{History of mTOR}

- Rapamycin is a bacterial product (Streptomyces hygroscopicus) found in soil samples from Rapa Nui (Easter Island).

- The first targets of rapamycin (Tor1, Tor2 and FKBP12) were identified in Saccharomyces cerevisiae.

- Eukaryotes have only one TOR gene, which is called mTOR.

\section{Multiprotein complexes of mTOR}

- mTOR forms two distinct multiprotein complexes: mTORC1 and mTORC2.

- The mTOR pathway has been linked to several disorders including cancer, diabetes, obesity, autoimmune disorders, neurological disease and aging

\section{Inhibitors of mTOR}

- Rapamycin (sirolimus) is a natural compound that has been approved in the clinical scenario as an immunosuppressant in kidney-transplanted patients and as an antiproliferative agent to prevent in-stent restenosis after angioplasty.

- The first-generation inhibitors of mTOR are analogs of rapamycin, also known as rapalogs, and include temsirolimus, everolimus and deforolimus.

- The second-generation inhibitors of mTOR are comprised of new compounds able to inhibit the ATP-binding site of mTORC1 and mTORC2, or PI3K and both mTORC1 and mTORC2.

\section{Molecular biomarkers in mTOR-targeted therapy}

- Numerous gaps still exist in our understanding of the molecular mechanisms underlying the response to mTOR inhibitors.

- There is an urgent need for efficient biomarkers not only to predict who will benefit from mTOR-based therapy but also to avoid the development of unnecessary toxicity.

\section{miRNAs \& $m$ TOR}

- miRNAs have been recently identified as new regulators of the mTOR-signaling pathway.

- miRNAs play a functional role in the response to mTOR inhibitors.

\section{References}

Papers of special note have been highlighted as:

- of interest

"I of considerable interest

1 Sehgal SN, Baker H, Vezina C. Rapamycin (AY-22,989), a new antifungal antibiotic. II. Fermentation, isolation and characterization. J. Antibiot. (Tokyo) 28(10), 727-732 (1975).

- Along with [2], this is one of the first original reports of the isolation of rapamycin.
2 Vezina C, Kudelski A, Sehgal SN. Rapamycin (AY-22,989), a new antifungal antibiotic. I. Taxonomy of the producing streptomycete and isolation of the active principle. J. Antibiot. (Tokyo) 28(10), 721-726 (1975).

- Along with [1], this is one of the first original reports of the isolation of rapamycin.

3 Galat A, Lane WS, Standaert RF, Schreiber SL. A rapamycin-selective $25-\mathrm{kDa}$ immunophilin. Biochemistry 31(8), 2427-2434 (1992).
4 Koltin Y, Faucette L, Bergsma DJ et al. Rapamycin sensitivity in Saccharomyces cerevisiae is mediated by a peptidyl-prolyl cis-trans isomerase related to human FK506binding protein. Mol. Cell. Biol. 11(3), 1718-1723 (1991)

5 Cafferkey R, Young PR, McLaughlin MM et al. Dominant missense mutations in a novel yeast protein related to mammalian phosphatidylinositol 3-kinase and VPS34 abrogate rapamycin cytotoxicity. Mol. Cell. Biol. 13(10), 6012-6023 (1993). 
6 Kunz J, Henriquez R, Schneider U, DeuterReinhard M, Movva NR, Hall MN. Target of rapamycin in yeast, TOR2, is an essential phosphatidylinositol kinase homolog required for G1 progression. Cell 73(3), 585-596 (1993).

7 Brown EJ, Albers MW, Shin TB et al. A mammalian protein targeted by G1arresting rapamycin-receptor complex. Nature 369(6483), 756-758 (1994).

" - Seminal paper reporting the identification of mTOR in eukaryotes.

8 Keith CT, Schreiber SL. PIK-related kinases: DNA repair, recombination, and cell cycle checkpoints. Science 270(5233), 50-51 (1995).

9 Chen J, Zheng XF, Brown EJ, Schreiber SL. Identification of an 11-kDa FKBP12rapamycin-binding domain within the 289$\mathrm{kDa}$ FKBP12-rapamycin-associated protein and characterization of a critical serine residue. Proc. Natl Acad. Sci. USA 92(11), 4947-4951 (1995).

10 Choi J, Chen J, Schreiber SL, Clardy J. Structure of the FKBP12-rapamycin complex interacting with the binding domain of human FRAP. Science 273(5272), 239-242 (1996).

11 Laplante M, Sabatini DM. mTOR signaling in growth control and disease. Cell 149(2), 274-293 (2012).

12 Takano A, Usui I, Haruta T et al. Mammalian target of rapamycin pathway regulates insulin signaling via subcellular redistribution of insulin receptor substrate 1 and integrates nutritional signals and metabolic signals of insulin. Mol. Cell. Biol. 21(15), 5050-5062 (2001).

13 Haruta T, Uno T, Kawahara J et al. A rapamycin-sensitive pathway downregulates insulin signaling via phosphorylation and proteasomal degradation of insulin receptor substrate-1. Mol. Endocrinol. 14(6), 783-794 (2000).

14 Sarbassov DD, Guertin DA, Ali SM, Sabatini DM. Phosphorylation and regulation of Akt/PKB by the rictor-mTOR complex. Science 307(5712), 1098-1101 (2005).

"- Authoritative paper showing for the first time that mTORC2 is able to activate Akt.

15 Iaccarino G, Ciccarelli M, Sorriento D et al. AKT participates in endothelial dysfunction in hypertension. Circulation 109(21), 2587-2593 (2004).

16 Huang J, Manning BD. A complex interplay between Akt, TSC2 and the two mTOR complexes. Biochem. Soc. Trans. 37(Pt 1), 217-222 (2009).

17 Teachey DT, Greiner R, Seif A et al. Treatment with sirolimus results in complete responses in patients with autoimmune lymphoproliferative syndrome. $B r$. J. Haematol. 145(1), 101-106 (2009).

18 Tsang CK, Qi H, Liu LF, Zheng XF. Targeting mammalian target of rapamycin (mTOR) for health and diseases. Drug Discov. Today 12(3-4), 112-124 (2007).

19 Marx SO, Jayaraman T, Go LO, Marks AR. Rapamycin-FKBP inhibits cell cycle regulators of proliferation in vascular smooth muscle cells. Circ. Res. 76(3), 412-417 (1995).

- Important study showing in vitro the relevance of rapamycin in the inhibition of vascular smooth muscle cell proliferation.

20 Morice WG, Brunn GJ, Wiederrecht G, Siekierka JJ, Abraham RT. Rapamycininduced inhibition of $\mathrm{p} 34 \mathrm{cdc} 2$ kinase activation is associated with G1/S-phase growth arrest in T lymphocytes. J. Biol. Chem. 268(5), 3734-3738 (1993).

21 Sarbassov DD, Ali SM, Sengupta S et al. Prolonged rapamycin treatment inhibits mTORC2 assembly and Akt/PKB. Mol. Cell 22(2), 159-168 (2006).

22 Poon M, Marx SO, Gallo R, Badimon JJ, Taubman MB, Marks AR. Rapamycin inhibits vascular smooth muscle cell migration. J. Clin. Invest. 98(10), 2277-2283 (1996).

23 Morris RE, Cao W, Huang X et al. Rapamycin (sirolimus) inhibits vascular smooth muscle DNA synthesis in vitro and suppresses narrowing in arterial allografts and in balloon-injured carotid arteries: evidence that rapamycin antagonizes growth factor action on immune and nonimmune cells. Transplant. Proc. 27(1), 430-431 (1995).

24 Gallo R, Padurean A, Jayaraman $\mathrm{T}$ et al. Inhibition of intimal thickening after balloon angioplasty in porcine coronary arteries by targeting regulators of the cell cycle. Circulation 99(16), 2164-2170 (1999).

- Significant and thorough study showing in vivo the importance of rapamycin in the inhibition of restenosis after angioplasty.

25 Atkins MB, Yasothan U, Kirkpatrick P. Everolimus. Nat. Rev. Drug Discov. 8(7), 535-536 (2009).

26 Krueger DA, Care MM, Holland K et al. Everolimus for subependymal giant-cell astrocytomas in tuberous sclerosis. N. Engl. J. Med. 363(19), 1801-1811 (2010).

27 Jozwiak S, Stein K, Kotulska K. Everolimus (RAD001): first systemic treatment for subependymal giant cell astrocytoma associated with tuberous sclerosis complex. Future Oncol. 8(12), 1515-1523 (2012).

28 Franz DN. Everolimus: an mTOR inhibitor for the treatment of tuberous sclerosis. Expert
Rev. Anticancer Ther. 11(8), 1181-1192 (2011)

29 Baselga J, Campone M, Piccart M et al. Everolimus in postmenopausal hormonereceptor-positive advanced breast cancer. N. Engl. J. Med. 366(6), 520-529 (2012).

30 O’Regan R. Phase III, randomized, double-blind, placebo-controlled multicenter trial of daily everolimus plus weekly trastuzumab and vinorelbine in trastuzumabresistant, advanced breast cancer (BOLERO-3). J. Clin. Oncol. 31, Suppl. Abstract 505 (2013).

31 Hudes G, Carducci M, Tomczak P et al. Temsirolimus, interferon alfa, or both for advanced renal-cell carcinoma. $N$. Engl. J. Med. 356(22), 2271-2281 (2007).

- Important clinical study demonstrating that the rapalog temsirolimus is able to improve overall survival among patients with metastatic renal-cell carcinoma.

32 Hess G, Herbrecht R, Romaguera J et al. Phase III study to evaluate temsirolimus compared with investigator's choice therapy for the treatment of relapsed or refractory mantle cell lymphoma. J. Clin. Oncol. 27(23), 3822-3829 (2009).

33 Murgia MG, Jordan S, Kahan BD. The side effect profile of sirolimus: a Phase I study in quiescent cyclosporine-prednisone-treated renal transplant patients. Kidney Int. 49(1), 209-216 (1996).

34 Karar J, Maity A. PI3K/AKT/mTOR pathway in angiogenesis. Front. Mol. Neurosci. 4, 51 (2011).

35 Ma WW, Adjei AA. Novel agents on the horizon for cancer therapy. CA Cancer J. Clin. 59(2), 111-137 (2009).

36 Guertin DA, Sabatini DM. The pharmacology of mTOR inhibition. Sci. Signal2(67), pe24 (2009).

37 Thoreen CC, Kang SA, Chang JW et al. An ATP-competitive mammalian target of rapamycin inhibitor reveals rapamycinresistant functions of mTORC1. J. Biol. Chem. 284(12), 8023-8032 (2009).

38 Choo AY, Yoon SO, Kim SG, Roux PP, Blenis J. Rapamycin differentially inhibits S6Ks and 4E-BP1 to mediate cell-type-specific repression of mRNA translation. Proc. Natl Acad. Sci. USA 105(45), 17414-17419 (2008).

39 Di Nicolantonio F, Arena S, Tabernero J et al. Deregulation of the PI3K and KRAS signaling pathways in human cancer cells determines their response to everolimus. J. Clin. Invest. 120(8), 2858-2866 (2010).

40 Carracedo A, Ma L, Teruya-Feldstein J et al. Inhibition of mTORC1 leads to MAPK pathway activation through a PI3K-dependent feedback loop in human cancer. J. Clin. Invest. 118(9), 3065-3074 (2008). 
- Noteworthy paper reporting the relevance of the mTORC1-MAPK feedback loop in human cancer.

41 Zhao H, Cui K, Nie F et al. The effect of mTOR inhibition alone or combined with MEK inhibitors on brain metastasis: an in vivo analysis in triple-negative breast cancer models. Breast Cancer Res. Treat. 131(2), 425-436 (2012).

42 Perino A, Ghigo A, Ferrero E et al. Integrating cardiac PIP3 and cAMP signaling through a PKA anchoring function of p110 $\gamma$. Mol. Cell 42(1), 84-95 (2011).

43 Delbaldo C, Albert S, Dreyer C et al. Predictive biomarkers for the activity of mammalian target of rapamycin (mTOR) inhibitors. Target Oncol. 6(2), 119-124 (2011).

44 Neshat MS, Mellinghoff IK, Tran C et al. Enhanced sensitivity of PTEN-deficient tumors to inhibition of FRAP/mTOR. Proc. Natl Acad. Sci. USA 98(18), 10314-10319 (2001).

45 Wang X, Yue P, Kim YA, Fu H, Khuri FR, Sun SY. Enhancing mammalian target of rapamycin (mTOR)-targeted cancer therapy by preventing $\mathrm{mTOR} /$ raptor inhibitioninitiated, mTOR/rictor-independent Akt activation. Cancer Res. 68(18), 7409-7418 (2008).

46 Witzig TE, Geyer SM, Ghobrial I et al. Phase II trial of single-agent temsirolimus (CCI-779) for relapsed mantle cell lymphoma. J. Clin. Oncol. 23(23), 5347-5356 (2005).

47 Guba M, von Breitenbuch P, Steinbauer M et al. Rapamycin inhibits primary and metastatic tumor growth by antiangiogenesis: involvement of vascular endothelial growth factor. Nat. Med. 8(2), 128-135 (2002).

48 Aguirre D, Boya P, Bellet D et al. Bcl-2 and CCND1/CDK4 expression levels predict the cellular effects of mTOR inhibitors in human ovarian carcinoma. Apoptosis 9(6), 797-805 (2004).

49 Totary-Jain H, Sanoudou D, Dautriche CN, Schneller H, Zambrana L, Marks AR. Rapamycin resistance is linked to defective regulation of Skp2. Cancer Res. 72(7), 1836-1843 (2012).

50 Mendell JT, Olson EN. MicroRNAs in stress signaling and human disease. Cell 148(6), 1172-1187 (2012).

51 Shomron N. MicroRNAs and pharmacogenomics. Pharmacogenomics 11(5), 629-632 (2010).

52 Cavarretta E, Chiariello GA, Condorelli G. Platelets, endothelium, and circulating microRNA-126 as a prognostic biomarker in cardiovascular diseases: per aspirin ad astra. Eur. Heart J. (2013) (Epub ahead of print).
53 Totary-Jain H, Sanoudou D, Ben-Dov IZ et al. Reprogramming of the microRNA transcriptome mediates resistance to rapamycin. J. Biol. Chem. 288(9), 6034-44 (2013).

- Original report identifying miRNAs as new downstream components of the mTOR signaling pathway.

54 Totary-Jain H, Marks AR. MicroRNAs and the cellular response to rapamycin: potential role in diagnosis and therapy. Cell Cycle 12(6), 861-862 (2013).

55 Li C, Liu Y, Liu J et al. Rapamycin inhibits human glioma cell proliferation through downregulating mammalian target of rapamycin pathway and up-regulating microRNA-143. Head Neck Oncol. 4(3), 66 (2012).

56 Liu L, Gong L, Zhang Y, Li N. Glycolysis in Panc-1 human pancreatic cancer cells is inhibited by everolimus. Exp. Ther. Med. 5(1), 338-342 (2013).

57 Jin XL, Sun QS, Liu F et al. microRNA 21-mediated suppression of Sproutyl by Pokemon affects liver cancer cell growth and proliferation. J. Cell. Biochem. 114(7), 1625-1633 (2013).

58 Sun Y, Ge Y, Drnevich J, Zhao Y, Band M, Chen J. Mammalian target of rapamycin regulates miRNA-1 and follistatin in skeletal myogenesis. J. Cell. Biol. 189(7), 1157-1169 (2010).

59 Ge Y, Sun Y, Chen J. IGF-II is regulated by microRNA-125b in skeletal myogenesis. J. Cell. Biol. 192(1), 69-81 (2011).

60 Fang Y, Xue JL, Shen Q, Chen J, Tian L. MicroRNA-7 inhibits tumor growth and metastasis by targeting the phosphoinositide 3-kinase/Akt pathway in hepatocellular carcinoma. Hepatology 55(6), 1852-1862 (2012).

61 Cui L, Zhou H, Zhao H et al. MicroRNA-99a induces G1-phase cell cycle arrest and suppresses tumorigenicity in renal cell carcinoma. BMC Cancer 12, 546 (2012).

62 Yan B, Fu Q, Lai L et al. Downregulation of microRNA 99a in oral squamous cell carcinomas contributes to the growth and survival of oral cancer cells. Mol. Med. Rep. 6(3), 675-681 (2012).

63 Wang Y, Liu J, Liu C, Naji A, Stoffers DA. MicroRNA-7 regulates the mTOR pathway and proliferation in adult pancreatic $\beta$-cells. Diabetes 62(3), 887-895 (2013).

64 Oneyama C, Ikeda J, Okuzaki D et al. MicroRNA-mediated downregulation of mTOR/FGFR3 controls tumor growth induced by Src-related oncogenic pathways. Oncogene 30(32), 3489-3501 (2011).

65 Iwaya T, Yokobori T, Nishida $\mathrm{N}$ et al. Downregulation of miR-144 is associated with colorectal cancer progression via activation of mTOR signaling pathway. Carcinogenesis 33(12), 2391-2397 (2012).

66 Uesugi A, Kozaki K, Tsuruta T et al. The tumor suppressive microRNA miR-218 targets the mTOR component Rictor and inhibits AKT phosphorylation in oral cancer. Cancer Res. 71(17), 5765-5778 (2011).

67 Grundmann S, Hans FP, Kinniry S et al. MicroRNA-100 regulates neovascularization by suppression of mammalian target of rapamycin in endothelial and vascular smooth muscle cells. Circulation 123(9), 999-1009 (2011).

68 Nagaraja AK, Creighton CJ, Yu Z et al. A link between miR-100 and FRAP1/mTOR in clear cell ovarian cancer. Mol. Endocrinol. 24(2), 447-463 (2010).

69 Wang FZ, Weber F, Croce C, Liu CG, Liao $X$, Pellett PE. Human cytomegalovirus infection alters the expression of cellular microRNA species that affect its replication. J. Virol. 82(18), 9065-9074 (2008).

70 Doghman M, El Wakil A, Cardinaud B et al. Regulation of insulin-like growth factormammalian target of rapamycin signaling by microRNA in childhood adrenocortical tumors. Cancer Res. 70(11), 4666-4675 (2010).

71 Sun J, Chen Z, Tan X et al. MicroRNA99a/100 promotes apoptosis by targeting mTOR in human esophageal squamous cell carcinoma. Med. Oncol. 30(1), 411 (2013).

72 Merkel O, Hamacher F, Laimer D et al. Identification of differential and functionally active miRNAs in both anaplastic lymphoma kinase (ALK)+ and ALK- anaplastic large-cell lymphoma. Proc. Natl Acad. Sci. USA 107(37), 16228-16233 (2010).

73 Fornari F, Milazzo M, Chieco P et al. MiR-199a-3p regulates mTOR and c-Met to influence the doxorubicin sensitivity of human hepatocarcinoma cells. Cancer Res. 70(12), 5184-5193 (2010).

74 Bhagwat SV, Gokhale PC, Crew AP et al. Preclinical characterization of OSI-027, a potent and selective inhibitor of mTORC1 and mTORC2: distinct from rapamycin. Mol. Cancer Ther. 10(8), 1394-1406 (2011).

75 Gupta M, Hendrickson AE, Yun SS et al. Dual mTORC1/mTORC2 inhibition diminishes Akt activation and induces Pumadependent apoptosis in lymphoid malignancies. Blood 119(2), 476-487 (2012).

76 Lewis GP, Chapin EA, Byun J, Luna G, Sherris D, Fisher SK. Muller cell reactivity and photoreceptor cell death are reduced after experimental retinal detachment using an inhibitor of the Akt/mTOR pathway. Invest. Ophthalmol. Vis. Sci. 50(9), 4429-4435 (2009). 
- Excellent paper showing that the simultaneous targeting of mTORC1 and mTORC2 may represent an effective antilymphoma strategy.

77 Chresta CM, Davies BR, Hickson I et al. AZD8055 is a potent, selective, and orally bioavailable ATP-competitive mammalian target of rapamycin kinase inhibitor with in vitro and in vivo antitumor activity. Cancer Res. 70(1), 288-298 (2010).

78 Willems L, Chapuis N, Puissant A et al. The dual mTORC1 and mTORC2 inhibitor AZD8055 has anti-tumor activity in acute myeloid leukemia. Leukemia 26(6), 1195-1202 (2012).

79 Houghton PJ, Gorlick R, Kolb EA et al. Initial testing (stage 1) of the mTOR kinase inhibitor AZD8055 by the pediatric preclinical testing program. Pediatr. Blood Cancer 58(2), 191-199 (2012).

80 Naing A, Aghajanian C, Raymond E et al. Safety, tolerability, pharmacokinetics and pharmacodynamics of AZD8055 in advanced solid tumours and lymphoma. Br. J. Cancer 107(7), 1093-1099 (2012).

81 Garcia-Garcia C, Ibrahim YH, Serra V et al. Dual mTORC1/2 and HER2 blockade results in antitumor activity in preclinical models of breast cancer resistant to anti-HER 2 therapy. Clin. Cancer. Res. 18(9), 2603-2612 (2012).

82 Pike KG, Malagu K, Hummersone MG et al. Optimization of potent and selective dual
mTORC1 and mTORC2 inhibitors: the discovery of AZD8055 and AZD2014. Bioorg. Med. Chem. Lett. 23(5), 1212-1216 (2013).

83 Maira SM, Finan P, Garcia-Echeverria C. From the bench to the bed side: PI3K pathway inhibitors in clinical development. Curr. Top. Microbiol. Immunol. 347, 209-239 (2010).

84 Venkatesan AM, Dehnhardt CM, Delos Santos E et al. Bis(morpholino-1,3,5-triazine) derivatives: potent adenosine 5 '-triphosphate competitive phosphatidylinositol-3-kinase/ mammalian target of rapamycin inhibitors: discovery of compound 26 (PKI-587), a highly efficacious dual inhibitor. J. Med. Chem. 53(6), 2636-2645 (2010).

85 Ghadimi MP, Lopez G, Torres KE et al. Targeting the PI3K/mTOR axis, alone and in combination with autophagy blockade, for the treatment of malignant peripheral nerve sheath tumors. Mol. Cancer Ther. 11(8), 1758-1769 (2012).

86 Yuan J, Mehta PP, Yin MJ et al. PF04691502, a potent and selective oral inhibitor of PI3K and mTOR kinases with antitumor activity. Mol. Cancer Ther. 10(11), 2189-2199 (2011).

87 Maira SM, Stauffer F, Brueggen J et al. Identification and characterization of NVPBEZ235, a new orally available dual phosphatidylinositol 3-kinase/mammalian target of rapamycin inhibitor with potent in vivo antitumor activity. Mol. Cancer Ther. 7(7), 1851-1863 (2008).

88 Chiarini F, Grimaldi C, Ricci F et al. Activity of the novel dual phosphatidylinositol 3-kinase/mammalian target of rapamycin inhibitor NVP-BEZ235 against T-cell acute lymphoblastic leukemia. Cancer Res. 70(20), 8097-8107 (2010).

89 Cerniglia GJ, Karar J, Tyagi S et al. Inhibition of autophagy as a strategy to augment radiosensitization by the dual phosphatidylinositol 3-kinase/mammalian target of rapamycin inhibitor NVP-BEZ235. Mol. Pharmacol. 82(6), 1230-1240 (2012).

90 Thomas HE, Mercer CA, Carnevalli LS et al. mTOR inhibitors synergize on regression, reversal of gene expression, and autophagy in hepatocellular carcinoma. Sci. Transl. Med. 4(139), 139ra184 (2012).

91 Figlin RA, Kaufmann I, Brechbiel J. Targeting PI3K and mTORC2 in metastatic renal cell carcinoma: new strategies for overcoming resistance to VEGFR and mTORC1 inhibitors. Int. J. Cancer 133(4), 788-796 (2013).

\section{Website}

101 Clinicaltrials.gov. http://clinicaltrials.gov 